\title{
CONTROLE DAS PLANTAS DAN INHAS EM CULTURA DE SOJA (Glycine max L. Merril) COM MISTURAS DE HERBICIDAS
}

\author{
L. S. P. CRUZ * \& L. LEIDERMAN * * \\ * Engo Agro Pesquisador Científico. Bolsis ta do \\ CNPq. \\ **Eng ${ }^{0}$ Agr $^{0}$ Pesquisador Científico Chefe \\ Seção de Herbicidas - Instituto Biológico \\ 13.100 - Campinas SP. \\ Trabalho apresentado na 29.a Reunião Anual \\ da SBPC, São Paulo, 1977. \\ Recebido para publicação em 12 de setembro \\ de 1977.
}

\section{RESUMO}

Em ensaio de campo, onde as principais plantas daninhas eram representadas por duas gramineas e seis ervas de folhas largas, foi comparada a ação de misturas de trifluralin, pendime thalin, dinitramine e alachlor com metribuzin e de alachlor com linuron, no seu controle.

Os resultados mostraram que as misturas de alachlor a $2,00 \mathrm{~kg} /$ ha e metribuzin a $0,35 \mathrm{~kg} / \mathrm{ha}$, e desses mesmos herbicidas a $3,00 \mathrm{~kg} / \mathrm{ha}$ e 0,50 $\mathrm{kg} / \mathrm{ha}$, feitas no tanque, aplicadas em pré-emergência, seguidas da mistura de trifluralin a 1,00 $\mathrm{kg} / \mathrm{ha}$, aplicado em pré-plantio incorporado, com metribuzin a $0,50 \mathrm{~kg} / \mathrm{ha}$, aplicado em pré-emergência, foram as mais eficientes.

Acanthospermum australe foi a planta da ninha de mais dificil controle. Somente a mistu ra de tanque de alachlor a $2,00 \mathrm{~kg} / \mathrm{ha}$ e linuron a $0,75 \mathrm{~kg} /$ ha foi eficiente no seu controle.

As misturas de pendimenthalin a $1,30 \mathrm{~kg} / \mathrm{ha}$ e de dinitramine a $0,50 \mathrm{~kg} / \mathrm{ha}$, ambos com me tribuzin a $0,50 \mathrm{~kg} / \mathrm{ha}$, retardaram o crescimento inicial da soja, porém sem prejudicar o stand e a produção. Os demais tratamentos também não foram prejudiciais à produção dessa leguminosa.

Unitermos: Controle, plantas daninhas, soja, misturas de herbicidas.

\section{SUMMARY}

\section{SOYBEAN WEED CONTROL WITH MIXTURES OF HERBICIDES}

Different mixtures of metribuzin with trifluralin, prendimetalin, dinitramine or alachlor were compared in order to evaluate weed control efficiency on soybean crop.

Tank mixtures of $2,00 \mathrm{Kg} /$ ha of alachlor and $0.35 \mathrm{Kg} / \mathrm{ha}$ of metribuzin, or $3,00 \mathrm{Kg} / \mathrm{ha}$ of alachlor and $0,50 \mathrm{Kg} / \mathrm{ha}$ of metribuzin, and 1,00 $\mathrm{Kg} / \mathrm{ha}$ of trifluralin and $0,50 \mathrm{Kg} / \mathrm{ha}$ of metribuzin were the most efficient in controlling gras ses and dicotyledons.

Acanthospermum australe was the most difficult weed to be controled. Only alachlor at $2,00 \mathrm{Kg} / \mathrm{ha}$ plus linuron at $0,75 \mathrm{Kg} / \mathrm{ha}$ gave satisfactory results.

The mixtures of pendimethalin at $1,30 \mathrm{~K} / \mathrm{ha}$ or dinitramine at $0,50 \mathrm{Kg} /$ ha plus metribuzin at $0,50 \mathrm{Kg} / \mathrm{ha}$ were fitotoxic at the initial growth stage of soybeans, but did not affect stand or yield. The other mixtures did not show any fitotoxicity and did not affect stand or yield either.

Keywords: Weed control, soybeans, mixtu res of herbicides.

\section{INTRODUÇÃO}

A cultura da soja no Brasil é uma das que mais emprega produtos químicos em capina, tanto pela eficiência do método como por sua economia. Como é comum o aparecimento de plantas daninhas como mono e dicotiledôneas, de espécies as mais variadas, torna-se difícil para um único herbicida combatê-las. Procura-se então associar dois ou mais herbicidas para proporcionarem um eficiente controle do mato. A escolha dos herbicidas e épocas de aplicação empregadas neste trabalho foram baseadas em produtos que anteriormente se mostraram bons no controle de determinadas plantas daninhas em soja, aliados a outros, bons para diferentes grupos de ervas más. Trifluralin, aplic $\mathrm{a}^{\mathrm{d}} \mathrm{o}$ em pré-plantio incorporado, vem sendo empre gado com grande eficiência no controle de gramíneas. 
Assim é que, em experimentos conduzidos em 1965/66 nos EEUU, Moore et al (9) já mostravam a eficiência desse herbicida em algodão e soja. Recentemente têm sido experimentados com sucesso os graminicidas pendimethalin1 e dinitramine. Bons resultados de controle de monocotiledôneas por pendimethal in foram obtidos em trabalhos de Grassi \& Leiderman (4) em soja, de Victoria et al. (16) em cana-deaçúcar e de Silva et al (14) em feijão. Dinitramine mostrou-se eficiente no controle de gramíneas, e compatível com metribuzin, em ensaios conduzidos em Jaboticabal-SP, por Victoria et al. (17) .

Para o controle de dicotiledôneas tem se destacado alachlor $(7,10,13,15)$, metribuzin $(6,8,15)$ e linuron (11). Muitos autores têm evidenciado a porcentagem maior de plantas daninhas controladas pelas misturas desses herbicidas $(3,5,12,17)$.

Com a finalidade de se obter maiores informações com misturas e combinações dos herbicidas anteriormente citados, foi conduzido um experimento em 1976/77.

\section{MATERIAIS E MÉTODOS}

Em outubro de 1976 foi instalado um ensaio de campo em Cristais Paulista-SP, em área de solo latossolo vermelho-amarelo, fase arenosa (2) com $30 \%$ de argila e $2,4 \%$ de matéria orgânica, cultivado anteriormente com milho.

O delineamento experimental escolhido foi o de blocos ao acaso com 12 tratamentos e quatro repetições, para possibilitar a análise estatística do stand e da produção de soja, feita pelo método da variância. Cada parcela tinha uma área de 21,00 $\mathrm{m}^{2}(3,00 \mathrm{x} \quad 7,00 \mathrm{~m})$ abrangendo cinco linhas de soja espaçadas de $0.60 \mathrm{~m}$. A área útil de cada parcela era formada pelas três linhas centrais, descontados $0.50 \mathrm{~m}$ de cad a extremidade.

As misturas de tanque de trifluralin $(\alpha, \alpha$, $\alpha$ triflu oro-2,6-dinitro-N,N-dipropil-p-toluidina) e metribuzin (4-amino-6-t-butil-3- (metiltio)1,2,4 -triazina-s- $(4 \mathrm{H})$-one) a $1,00 \mathrm{~kg} \mathrm{e} 0,75$ $\mathrm{kg} / \mathrm{ha}$ e a $0,50 \mathrm{~kg}$ e $0,35 \mathrm{~kg} / \mathrm{ha}$, respectiva-

\footnotetext{
${ }^{1}$ Nome anteriormente proposto: penoxalin
}

mente, as sim como de pendimethalin (N - (1 etilpropil) -3,4-dimetil-2,6-di nitrobenzenoamina) a $1,30 \mathrm{~kg} / \mathrm{ha}$ e de dinitramine $\left(\mathrm{N}^{3} \mathrm{~N}^{3}\right.$ dietil -2,4dinitro-6-trif luorometil-m-fenilenediamina) a $0,50 \mathrm{~kg} / \mathrm{ha}$, ambos misturados com metri buzin a $0,50 \mathrm{~kg} / \mathrm{ha}$, foram aplicadas em pré plantio incorporado, 24 horas antes do plan tio de soja. Nesta mesma ocasião, trifluralin a $1.00 \mathrm{~kg} / \mathrm{ha}$ e a $0,75 \mathrm{~kg} / \mathrm{h}$ a ta mbém foi apli cado isoladamente e incorporado ao solo com grade de discos de 18" trabalhando a uma profundidade mé dia de $0,12 \mathrm{~m}$. Logo apó $\mathrm{s}$ o plantio da soja, a estes do is últimos t ratamentos foi adicionada uma aplicação pré emergente de metribuzin a $0,50 \mathrm{~kg} / \mathrm{ha}$ e a $0.35 \mathrm{~kg} / \mathrm{ha}$, respectivamente. Nesta data tam bém foram aplicados em pré-emergência, em mistura de tanque: alachlor (2 -cloro-2'.6'-dietil-N- (metoximetil) -acetanilida) a $3,00 \mathrm{~kg} / \mathrm{ha}$ com metribuzin a $0,50 \mathrm{~kg} / \mathrm{ha}$; alachlor a 2,00 $\mathrm{kg} / \mathrm{ha}$ com metribuzin a $0,35 \mathrm{~kg} / \mathrm{ha}$, e, ala chlor a $2,00 \mathrm{~kg} / \mathrm{ha}$ com linuron (3 - (3,4-diclorofenil) -1-metoxi-1-metiluréia) a $0,75 \mathrm{~kg} / \mathrm{ha}$. Foi incluido ainda um tratamento somente com al achlor a 3,00 kg/h a, aplicado em préemergência, pulverizado também no mesmo dia do plantio de soja.

As aplicações foram feitas com pulverizadores tostai s, com ca pacidade para $14 \mathrm{li}$ tros, manuais, com agitad or de calda, muni dos de um bico de jato em leque 80.03, com peneira de malha 50, trabalhando a $40 \mathrm{lb}$ de pressão/pol ${ }^{2}$, com um gasto correspondente a 400 litros de calda herbicida por hectare.

Constaram ainda do ensaio do is tratamentos testemunhas, sem herbicida, um mantido sempre no limpo e outro, sem capina até a colheita.

A variedade de soja usada foi a IAC-2. inoculada, tendo sido gastas 25 sementes por metro de sulco.

Os corretivos e os adubos químicos foram aplicados de acordo com os resultados das análises de solo. O ensaio foi mantido em boas condições de sanidade com aplicações de inseticidas, iguais para todos os tratamentos.

Para a avaliação da eficiência das misturas de herbicidas foi considerada a porcentagem de controle das plantas infestantes tomada sempre em re lação à testemunha sem herbicida. Essa porcentagem de controle foi obtida de amostragem do número de plantas daninhas incidentes nas parcelas tratadas e na 
te stemunha, to mando-se duas amostras de $0,50 \mathrm{~m}^{2}(1,00 \mathrm{~m} \times 0,50 \mathrm{~m})$ por parcela. após 40 dias. Aos 40, 60 e 90 dias da aplicação dos produtos foram feitas observações visuais de infestação existente no ensaio.

A infestação natural das principais plantas daninhas era representada por: capim-decolchão (Digitaria sanguinalis (L) Scop.) , ca pim-pé-de-galinha (Eleusine indica (L) Gaertn) , poaia-branca (Richardia brasiliensis Gomez) , picão-preto (Bidens pilosa L) , carrapicho-do-campo (Acanthospermum australe (Loef) O. Kuntze), gu an xuma (Sida rhombifolia L), falsa-serralha (Emilia sonchifolia DC) e beldroega (Portulacca oleracea L).

Foram realizadas observações visuais s obre sintomas característicos de fitotoxicidade causados pelos herbicidas sobre a cultura, 20 dias após o plantio. Foram consideradas notas de 1 (soja totalmente prejudicada) a 5 (soja sem fitotoxicidade). Nesta data também foi an otado o stand da cultura. A colheita foi realizada a 29 de maio de 1977.

\section{MATERIAIS E MÉ TODO}

Na tabela 1 são representadas as porcentagens de controle de plantas daninhas, assim como as notas dadas para sintomas de fitotoxicidade, as médias de número de plantas (stand) trans formados em RQ (x) e as mé dias dos dados originais de produção de soja, em quilos por tratamento.

As duas gramíneas incidentes e ainda $R$. brasiliensis, B. pilosa, E. sonchifolia, $S$. rhombifolia e $P$. oleracea foram bem controladas por todas as misturas de herbicidas, e também por alachlor quando empregado isoladamente. A. australe foi bem controlado apenas por alachlor + linuron.

No controle geral de plantas daninhas os melhores resultados foram obtidos com as misturas de alachlor metribuzin a $2,00 \mathrm{~kg}$ $+0,35 \mathrm{~kg} / \mathrm{ha}$ e a $3,00 \mathrm{~kg}-1-0,50 \mathrm{~kg} / \mathrm{ha}$ aplicados em pré-emergência, e com a mistura de triflural in a $1,00 \mathrm{~kg} / \mathrm{ha}$, em pré-plantio incorporado, com metribuzin a $0,50 \mathrm{~kg} / \mathrm{ha}$, em pré-emergência, todas com índices superiores a $94 \%$ de controle. Metribuzin ap resentou melhores resultados quando aplicado em pré-emergência, comparado com pré-plantio incorporado, sempre que aliado com triflu ralin em pré-plantio incorporado. Borgo \& Beskow (1) também encontraram resultados melhores para metribuzin aplicado na superfície.

Alachlor, a 3,00 kg/ha, em pré-emergência, somente não conseguiu controlar A. australe, com um índice de controle geral de 92\% Este resultado difere do encontrado por Borgo \& Beskow (1) em ensaios conduzid os em 1974/75 e 1975/76, onde alachlor em aplicação isolada foi bastante inferior às misturas testadas . Convém salientar que esses resultados referemse a experimento em solo com mais de $4 \%$ de matéria orgânica, e -com incidência de outras plantas daninhas.

As misturas de pendimethalin e de dinitramine com metribuzin apre sentaram sintomas de fito toxici dade quando da observação aos 20 dias do plantio de soja. Esses sintomas foram caracterizados pela mudança de coloração das folhas e retardamento do desenvolvimento vegetativo inicial da leguminosa. Eles desapareceram depois e as análises estatísticas do stand e da produção não mostraram diferenças significativas entre os tratamentos. Victoria et al. (16) também não encontraram diferenças de produção para dinitramine + metribuzin a $0,50 \mathrm{~kg}+0,50 \mathrm{~kg} / \mathrm{ha}$, em ensaio conduzido em latossolo vermelho escuro, fase arenosa, com $2,3 \%$ de matéria orgânica.

Todos os tratamentos com herbicidas não precisaram de capina adicional até a colheita. A testemunha que deveria ser mantida sempre no limpo foi capinada aos 20 e aos 60 dias do plantio de soja, e na colheita. $\mathrm{Na}$ observação visual feita aos 60 dias, a testemunha sem capina apresentava uma infestação média de $68 \%$ e aos 90 dias, de $81 \%$. Nesta data, o tratamento de alachlor + linuron a $2,00 \mathrm{~kg}+$ $0,75 \mathrm{~kg} / \mathrm{ha}$ tinha a menor porcentagem de infestação de plantas daninhas seguido do tratame nto alachlor + metribuzin, a 2,00 kg + $0,35 \mathrm{~kg} / \mathrm{ha}$.

\section{AGRADECIMENTO}

Ao Sr. José Alexandre Junqueira Vilella, proprietário da Fazenda Belo Horizonte, onde foi realizado o experimento. 
Tabela 1 - Porcentagem de controle de plantas daninhas (1), fitotoxicidade (2), média de stand com dados transformados para $\sqrt{\mathrm{x}} \mathrm{e}$ média de dados originais de produçāo de soja em Cristais Paulista, SP, 1976/77. Os dados são médias de quatro repetiçôes.

\begin{tabular}{|c|c|c|c|c|c|c|c|c|c|c|c|c|c|c|}
\hline \multirow{2}{*}{\multicolumn{2}{|c|}{ TPATAMENTO }} & \multirow{2}{*}{$\begin{array}{c}\text { Ingredien- } \\
\text { te ativo } \\
\text { (kg/ha) }\end{array}$} & \multicolumn{9}{|c|}{ PORCENTAGEM DE CONTROLE } & \multirow{2}{*}{$\begin{array}{l}\text { Fitoto- } \\
\text { xici- } \\
\text { cidade }\end{array}$} & \multirow{2}{*}{ stand } & \multirow{2}{*}{$\begin{array}{l}\text { Produ- } \\
\text { ç্⿳亠口冋o }\end{array}$} \\
\hline & & & $D \operatorname{san}^{(3)}$ & $\mathrm{E}$ ind ${ }^{(3)}$ & R bra ${ }^{(3)}$ & B pil $^{(3)}$ & $A$ aus ${ }^{(3)}$ & $\mathrm{S}$ rho & 5 $\operatorname{son}^{(3)}$ & Pole ${ }^{(3)}$ & C ger $^{(3)}$ & & & \\
\hline Trifluralin (4) & + metribuzin (6) & $1.00+0.50$ & 96,7 & 100,0 & 96,0 & 87.2 & 51,8 & 76.9 & 91.7 & 100,0 & 91,5 & 5 & 12.439 & 1.270 \\
\hline Trifluralin (4) & + metribuxin (4) & $0.75+0.35$ & 99.4 & 95,0 & 90.7 & 83.8 & 53,6 & 84.6 & 100.0 & 100.0 & 88.8 & 5 & 13,055 & 1,041 \\
\hline Trifluralin (4) & + metribuzin (5) & $1,00+0.50$ & 99,3 & 100,0 & 97.3 & 99.1 & 50.0 & 88.5 & 91.7 & 100,0 & 94.8 & 5 & 12,529 & 1,092 \\
\hline Trifluralin (4) & $f$ metribuzin (s) & $0.75+0.35$ & 99.3 & 99,0 & 96.0 & 91,4 & 1.8 & 100.0 & 100.0 & 100,0 & 90.3 & 5 & 13,082 & 1,188 \\
\hline Alnchlor (5) & + metribuzin (5) & $3.00+0.50$ & 97.1 & 160,0 & 96.0 & 97,4 & 60,7 & 100.0 & 100,0 & 100.0 & 94.9 & 5 & 13,476 & 1,206 \\
\hline Alachlor (5) & + metribuzin (5) & $2.00+0.35$ & 97,4 & 100,0 & 96,0 & 99,1 & 58.9 & 100,0 & 100.0 & 100.0 & 95,2 & 5 & 13,113 & 1,000 \\
\hline Alachlor (5) & +1 inuron (5) & $2.00+0.75$ & 96.0 & 100.0 & 97.3 & 79.5 & 85.7 & 96,1 & 100,0 & 100.0 & 92.7 & 5 & 13,528 & 1.195 \\
\hline Alachlor (5) & & 3.00 & 94.5 & 94.0 & 95,4 & 100,0 & 46,4 & 88.5 & 100.0 & 100,0 & 92.0 & 5 & $12.9 \mathrm{M}$ & 0,988 \\
\hline Pendimethalin (4) & + metribuzin (4) & $1.30+0.50$ & 100,0 & 98.0 & 98,7 & 92,3 & 32.1 & 92.3 & 95.8 & 100,0 & 92.9 & 3 & 12,646 & 0.956 \\
\hline Dinitramine (4) & + mot ibuzin $(4)$ & $0.50+0.50$ & 98,5 & 98.0 & 96,0 & 75.2 & 0.0 & 80.8 & 95.8 & 100,0 & 86.5 & 4 & 13.098 & 1,012 \\
\hline \multicolumn{2}{|c|}{ Testemunha capinada } & - & $\longrightarrow$ & $\longrightarrow$ & $\longrightarrow$ & $\longrightarrow$ & $\longrightarrow$ & $\longrightarrow$ & $\longrightarrow$ & $\longrightarrow$ & & 5 & 12,926 & 1,062 \\
\hline \multicolumn{2}{|c|}{ Testemunha sem capina } & $\cdots$ & $\longrightarrow$ & $\longrightarrow$ & $\longrightarrow$ & $\longrightarrow$ & $\longrightarrow$ & $一$ & $\longrightarrow$ & $\longrightarrow$ & $\longrightarrow$ & 5 & 12,350 & 0,797 \\
\hline \multicolumn{2}{|c|}{ Testemunha (n.\% médio de pts. dan./m²) } & & 275 & 101 & 151 & 117 & 56 & 26 & 24 & 17 & 767 & $\rightarrow$ & $\longrightarrow$ & - \\
\hline
\end{tabular}

$\begin{array}{lrr}\text { C.V.(\%) } & 8.06 & 19.72 \\ \text { F. } & 0.52 & 1.57\end{array}$

(1) 40 dias após a aplicação dos herbicidas.

(2) 20 dias após a aplicação dos herbicidas.

(3) Abreviaturas usadas: D san - Digitaria sanguinalis, E ind - Eleusine indica, R bra - Richardia brasiliensis, B pil Bidens pilosa, A aus - Acanthospermum australe, S rho - Sida rhombifolia, E son-Emilia sonchifolia, $\mathrm{P}$ ole -- Portulacca oleracea e C ger - Controle geral.

(4) Aplicado em pré-plantio incorporado.

(5) Aplicado em pré-emergência. 


\section{LITERATURA CITADA}

1. BORGo, A. \& BESKow, G. Testes de herbi. cidas combinados na cultura da soja. In: Sem. Bras. Herb. Ervas Danin., $11^{\circ}$, Lon. drina, 1976. Resumos, 88.

2. COMISSÃO DE SOLOS DO CENTRO NACIO. NAL DE ENSINO E PESQUISAS AGRO. NómICAS. Cartas dos solos do Estado de Săo Paulo. Bol. Serv. Nac. Pesq. Agric. Min. Agric. (12):114-36, 1960.

3. COVOLO, L. \& PULver, E. Ensaio de com. petição de herbicidas, em misturas de tan. que, na cultura da soja. In: Sem. Bras. Herb. Ervas Dan., 11\%, Londrina, 1976. Re. sumos, 78 .

4. GRASSI, N. \& LEIDERMAN, L. Dois novos herbicidas de pré-plantio incorporado na cultura da soja. In: Sem, Bras. Herb. Er. vas Dan., $11^{\circ}$, Londrina, 1976. Resumos, 28 .

5. JENNINGS, V.M. Soybean herbicide evalua. tions across Iowa in 1974. In: Proc. 29th N. Cent. Weed Control Conf., 1974, 29:79-83.

6. LORENZI, H.J. \& DAVIS, G.G. Competição de herbicidas na cultura da soja. In: Sem. Bras. Herb. Ervas Dan., 11', Londrina, 1976. Resumos, 67.

7. LOVELY, W.G. \& STANIFORTH, P.W. Sys. tems of weed control for soybean produc tion using variable row width. In: Proc. 23rd N. Cent. Weed Control Conf., 1968, 28.9.

8. MOOMAW, R.S. \& BURNSIDE, O.S. Soybean herbicide trials in Nebraska in 1972. In: Proc. 27th N. Cent. Weed Control Conf., $1972,27: 25-7$.

9. MOORE. C.E., BARRENTINE, J.L., ARNOLD, W.R. \& KEATON, J.A, Fall and winter aplication of trifluralin, a new concept in weed control. In: Meet. Weed Soc. Am. 1967. Abstract, 2-3.

10. RAmos, M. Controle químico de invasoras na cultura de soja-75/76. Bol. EMBRAPA 04, dezembro 1976, Ponta Grossa, 1976 $25 \mathrm{p}$.

11. RILEY, W.R. Herbicides on soybeans. Mac. donald Coll., Mc. Gill Univ,, Montreal, Ca. nadá. $101 \mathrm{p}$. Dissert.

12. ROBISON, L.R. Soybean herbicide perfor mance trials in Nebraska. In: Proc. 24th N. Cent. Weed Control Conf., 1969, 28-30.

13. SCUDDER, W.T. Chemical control of weeds in field crop. Res. Rep. Inst. Fd. Agric Sci., Florida, 1970. $134 \mathrm{p}$.

14. SILVA, J.F., SILVA, C.M., SEDIYAMA, C.S \& CosTA, L.M. Efeitos de alguns herbici das no controle de ervas daninhas na pro dução e qualidade fisiológica de semente: de feijoeiro. In: Sem. Bras. Herb. Ervas Dan., $11^{\circ}$, Londrina, 1976. Resumos, 55.

15. VENTURELA, L.R.C., RUCKHEIN Filho, O \& DAVIS, D.D. Herbicidas isolados no controle às ervas daninhas, da s'ja (Gly cine max. (L.) Merril). In: Sem. Bras Herb. Ervas Dan., 11\%, Londrina, 1976. Re sumos, 71 .

16. VICTORIA Filho, R., SILVA, J.A. \& SAN TOS, D.P. Herbicidas pré-emergentes n cultura de cana-de-açúcar (Saccharum effi cinarum L) In: Sem. Bras, Herb. Frva Dan., 11', Londrina, 1976. Resumos, 56.

17. VICTORIA Filho, R., GARCIA, I. \& CRUZ L.S.P. Controle de plantas daninhas na cul tura de soja (Glyeine max (L) Merril com herbicidas em pré-plantio incorpora do. In: Sem. Bras. Herb. Ervas Dan., 11* Londrina, 1976. Resumos, 73. 